\title{
High-extraction-efficiency operation of a midinfrared free electron laser enabled by dynamic cavity desynchronization
}

\author{
Heishun Zen๑* and Hideaki Ohgakiఠ \\ Institute of Advanced Energy, Kyoto University, Gokasho, Uji, Kyoto 6110011 Japan \\ Ryoichi Hajima $\odot$ \\ National Institutes for Quantum and Radiological Science and Technology, \\ Tokai, Naka, Ibaraki 3191106 Japan
}

(Received 13 April 2020; accepted 17 June 2020; published 6 July 2020)

\begin{abstract}
The extraction efficiency is one of the key parameters of an oscillator-type free electron laser (FEL). An extraction efficiency higher than 5\% was observed with the midinfrared (MIR) FEL at Kyoto University using a dynamic cavity desynchronization method. The observed efficiency is the highest extraction efficiency realized in MIR-FEL oscillators driven by normal conducting linacs. A numerical simulation was performed, and the evolution of the electron energy distribution in the macropulse was compared with the experimental result. The details of the evolution of the electron energy distribution were investigated to reveal the physics of this high-efficiency lasing.
\end{abstract}

DOI: 10.1103/PhysRevAccelBeams.23.070701

\section{INTRODUCTION}

The oscillator-type midinfrared free electron laser (MIRFEL) is one of the intense tunable coherent radiation sources in the MIR wavelength region. After the first lasing of the FEL at $3.4 \mu \mathrm{m}$ [1], many MIR-FELs have been constructed and used in a broad range of applications [2-16]. A FEL converts the kinetic energy of an electron beam into an energy of the electromagnetic wave through FEL interaction in undulators. The energy conversion efficiency, called the extraction efficiency, is one of the key parameters of the FEL that determines the peak power and pulse duration of oscillator-type FELs. The world's highest extraction efficiency of an oscillator-type FEL, achieved with the Japan Atomic Energy Research Institute FEL (JAERI-FEL) [17], is 9\% at a wavelength of $22 \mu \mathrm{m}$. This high-efficiency condition was achieved by the perfect synchronization of the electron beam repetition rate with the optical cavity roundtrip frequency. The JAERI-FEL used a superconducting (SC) accelerator with a uniform undulator. A long-duration macropulse $(\sim 1 \mathrm{~ms})$ from the SC accelerator [18] enabled FEL lasing under the perfect synchronization condition. An alternative method to increase the extraction efficiency is the undulator tapering, i.e., gradually reducing the magnetic field strength along

\footnotetext{
zen@iae.kyoto-u.ac.jp
}

Published by the American Physical Society under the terms of the Creative Commons Attribution 4.0 International license. Further distribution of this work must maintain attribution to the author(s) and the published article's title, journal citation, and DOI. the undulator [19]. The use of a tapered undulator can increase the extraction efficiency of the single-pass amplifier. For example, the UCLA-BNL-TelAvivUniv. group achieved an extraction efficiency of $30 \%$ by using a strongly tapered undulator and injecting a high-power $\mathrm{CO}_{2}$ laser [20]. Undulator tapering is also effective in oscillator-type FELs; an extraction efficiency of $4.4 \%$ at a wavelength of $11 \mu \mathrm{m}$ was achieved with the LANL FEL [21]. A further enhancement of the extraction efficiency in an FEL oscillator was recently proposed with a combination of a strongly tapered undulator and a seed laser for prebunching [22].

In this paper, we report the high-efficiency operation of an MIR-FEL named the Kyoto University FEL (KU-FEL), which is enabled by the dynamic cavity desynchronization (DCD) technique invented by the FELIX group [23]. The extraction efficiency is evaluated from the evolution of the electron energy distribution measured at the undulator exit with and without FEL lasing. The DCD parameters are varied to confirm the effectiveness of DCD. A numerical simulation is also carried out to reveal the physics of this high-extraction-efficiency lasing.

\section{EXTRACTION EFFICIENCY OF AN OSCILLATOR-TYPE FEL AND ITS MEASUREMENT METHOD}

\section{A. Extraction efficiency of an oscillator-type FEL}

The extraction efficiency of an FEL, $\eta$ is defined as the fraction of the kinetic energy of the electron beam that is transferred to electromagnetic energy during the FEL interaction [24]: 


$$
\eta=\Delta W_{e} / W_{e 0},
$$

where $W_{e 0}=Q\langle\gamma\rangle\left(m_{0} c^{2} / e\right)$ is the total kinetic energy of the electron bunch before the FEL interaction, $Q$ is the charge of each electron bunch, $\gamma$ is the Lorentz factor of the electron, $m_{0}$ is the electron rest mass, $c$ is the velocity of light, $e$ is the elementary charge, and $\Delta W_{e}$ is the amount of energy transferred to electromagnetic energy in the FEL interaction from each electron bunch. The amount $\Delta W_{e}$ can be written as

$$
\Delta W_{e}=W_{e 0}-W_{e 1},
$$

where $W_{e 1}$ is the total electron bunch energy after the FEL interaction. Under the saturation condition, the energy loss in the optical cavity $W_{\text {loss }}$ and the extracted energy from the electron beam balance each other:

$$
W_{\text {loss }}=\Delta W_{e}=\eta W_{e 0} .
$$

The optical cavity loss can be divided into the energy outcoupling loss $W_{\text {out }}$, the reflection loss $W_{\text {ref }}$, and the diffraction loss $W_{\text {diff. }}$. The energy out-coupled from the cavity $W_{\text {out }}$ can be calculated by

$$
W_{\text {out }}=\frac{W_{\text {out }}}{W_{\text {out }}+W_{\text {ref }}+W_{\text {diff }}} W_{\text {loss }}=k(\lambda) \eta W_{e 0},
$$

where $k(\lambda)=W_{\text {out }} /\left(W_{\text {out }}+W_{\text {ref }}+W_{\text {diff }}\right)$ is the out-coupling loss ratio, which depends on the lasing wavelength $\lambda$, the optical cavity arrangements, and the transverse aperture of the beam duct.

Several theoretical studies have been carried out to discuss the limitations of the extraction efficiency of oscillator-type FELs driven by short-bunch electron beams $[25,26]$. Under the short bunch condition where the electron bunch length $L_{b}$ is shorter than the slippage length $L_{S}$, the extraction efficiency $\eta$ is determined by the superradiant scaling law of a short-bunch FEL oscillator [25,26]. The efficiency for small negative detuning condition is given by [25]

$$
\eta \sim \rho \sqrt{\frac{L_{b}}{\alpha_{0} L_{c}}},
$$

where $\rho$ is the fundamental FEL parameter [27], $L_{c}=$ $\lambda /(4 \pi \rho)$ is the cooperation length [28], and $\alpha_{0}$ is the total cavity loss. Under this condition, the FEL gain is reduced from the long electron bunch condition $\left(L_{b}>L_{S}\right)$, and the maximum efficiency available is larger than the long electron bunch condition $\eta \sim 1 /\left(2 N_{w}\right)$, where $N_{w}$ is the number of undulator periods. It has been reported that higher extraction efficiency than that given by Eq. (5) can be obtained under the perfectly synchronized cavity length condition $[18,26]$. For the experimental condition of present study, the extraction efficiency calculated with Eq. (5) is approximately $2.4 \%$. In addition, the FEL output pulse has a narrow spike of $N_{S} \sim 1 /(8 \pi \eta)$ optical periods. An FEL with a higher extraction efficiency can have a shorter pulse duration under the short electron bunch condition.

\section{B. Method for the extraction efficiency measurement}

Based on Eqs. (1) and (2), the extraction efficiency can be determined by measuring the variation in the average energy of the electron bunch with and without FEL lasing. In many facilities, this method has been used for the extraction efficiency measurement [18,21,24]. For the JAERI-FEL [18], the researchers evaluated the extraction efficiency from the out-coupled FEL power, which was calibrated by the electron beam energy spectrum under certain lasing conditions. In this case, the researchers combined Eqs. (1) and (4) for an easy and continuous measurement of the efficiency. Since the absolute FEL power and the out-coupling loss ratio $k(\lambda)$ cannot be accurately determined due to time varying spectrum of FEL and presence of optical components before a power meter, direct evaluation of the extraction efficiency from Eq. (4) has much less accuracy than that evaluated from the variation in the average energy of the electron bunch. Therefore, the researchers in JAERI-FEL performed calibration of $k(\lambda)$ at certain lasing condition. For the CLIOFEL [24], the researchers measured the temporal evolutions of the energy spectrum of the electron beam in a macropulse with and without FEL lasing. The method used for the CLIO-FEL provides time-dependent information on the FEL lasing dynamics. Therefore, we adopt this method for the extraction efficiency measurement in this study.

\section{DYNAMIC CAVITY DESYNCHRONIZATION [23]}

In an FEL oscillator driven by short electron bunches, the lasing dynamics are a function of the cavity detuning, which is realized by a shortening of the cavity length from the length synchronized to the electron bunch arrival period. The optimum cavity detuning used to obtain the shortest buildup time to saturation is different from that used to maximize the saturation power due to the slippage of the electron bunch and the optical pulse in the undulator [25]. The DCD method was invented to achieve the shortest buildup time and the largest saturated power at the same time [23]. In the DCD method, the synchronization condition between the electron bunch repetition rate and the roundtrip frequency of the optical cavity is dynamically varied within a macropulse by modulating the electron bunch repetition rate. The FELIX group clearly demonstrated that DCD can simultaneously realize an operating condition to enable fast buildup and high saturated power [23]. In the report, the achievable extraction efficiency 
using the DCD was not discussed. The FELIX group reported that the extraction efficiency was approximately $4 \%$ without DCD [29].

In 2000, Korean researchers reported the results of numerical simulations on DCD, including a discussion on the extraction efficiency [30]. The researchers showed that the extraction efficiency of a FEL oscillator can be clearly increased by using the DCD method in the case of the nominal parameter of FELIX. This simulation work predicted that the highest extraction efficiency achievable in the previous FELIX experiment was approximately $7.5 \%$. It was also shown that the DCD method is particularly useful for achieving a high extraction efficiency in oscillator type FELs driven by a normal conducting rf linac, which has a limited macropulse duration like the KU-FEL (macropulse duration of $6.5 \mu \mathrm{s}$ ).

\section{EXPERIMENTAL SETUP AND CONDITIONS}

\section{A. Setup of the extraction efficiency measurement}

The experimental setup of the extraction efficiency measurement is shown in Fig. 1. A bending magnet and a Faraday cup with an aluminum slit were used. The slit width and thickness were $3 \mathrm{~mm}$ and $60 \mathrm{~mm}$, respectively. The bending magnet used in this experiment was a sector magnet, which had no strong edge focusing effect like a rectangular magnet. The Faraday cup was connected to an oscilloscope to record the charge variation in a macropulse. To measure the energy distribution of the electron beam, the excitation current of the bending magnet was varied to change the energy of the electron that reached the Faraday cup after the slit. By stacking the measured charge variation in a macropulse with different excitation current of the bending magnet, we can obtain a time dependent energy distribution in macropulse. An intracavity beam shutter was installed between the bending magnet and a FEL resonator mirror. The beam shutter enables us to turn on and off the FEL lasing without changing any other condition. The energy resolution of the system was limited by the slit

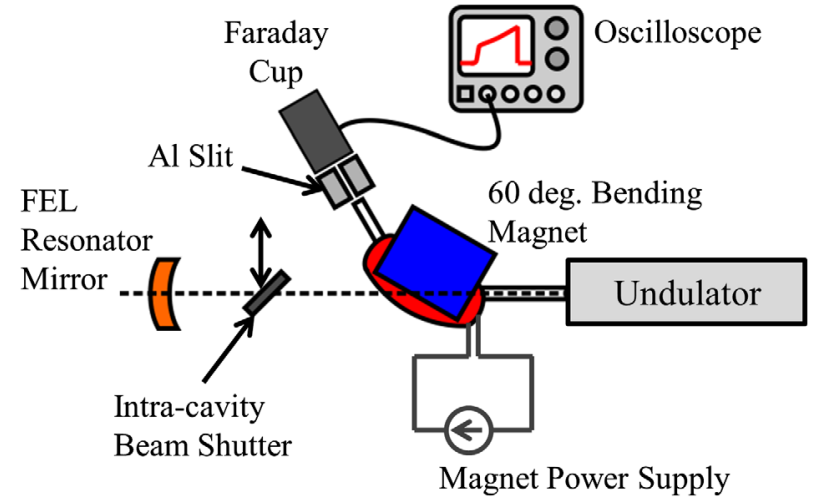

FIG. 1. Schematic drawing of the experimental setup for the extraction efficiency measurement. Only the downstream side of the undulator is depicted in this figure.

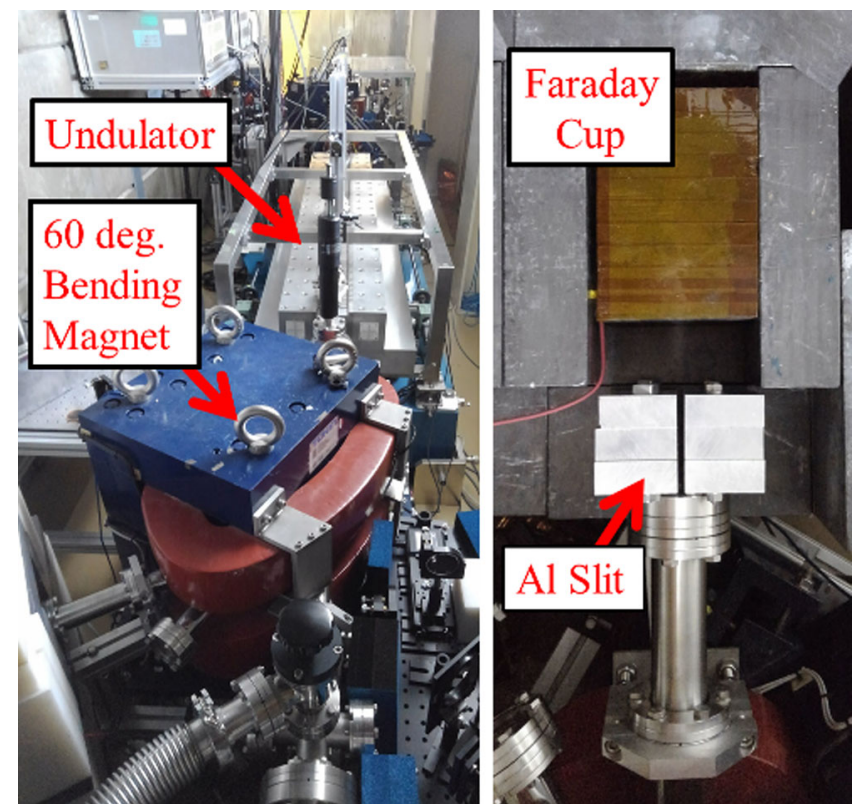

FIG. 2. Photographs of the Faraday cup, aluminum slit, bending magnet, and undulator.

width and the horizontal beam size at the entrance of the bending magnet. We expected an energy resolution of $1 \%$ in the experiment from the geometry and the electron beam size. Photographs of these components are shown in Fig. 2.

\section{B. Setup and conditions of DCD}

The electron bunch repetition rate was dynamically modulated in a macropulse to vary the synchronization condition between the electron bunch and the optical pulse in the FEL oscillator. A schematic diagram of the rf system used to drive the KU-FEL linac is shown in Fig. 3. The electron gun used in the KU-FEL is a 4.5-cell thermionic rf gun driven by a 2856-MHz klystron. In the case of the thermionic rf gun, the electron bunch repetition rate is determined by the frequency of the rf source. Two fast phase shifters (PS-3-2856, R\&K Co., Ltd.) were installed before klystron driver amplifiers. These fast phase shifters were originally installed to compensate for the rf phase variation in a macropulse. Using the phase shifters, we can realize the frequency modulation that is necessary for DCD as follows:

$$
V(t)=A \cos (\omega t+a t)=A \cos \{(\omega+a) t\},
$$

where $V(t)$ is the rf voltage, and $a$ is a linear phase shift coefficient and $\omega$ is the angular frequency. In our experiment, we used a function generator to realize a phase shift with an arbitrary waveform.

Another important component for DCD is the bunch phase monitor used to measure the variation in the electron bunch repetition rate. Based on Eq. (6), the variation in the electron bunch repetition rate can be calculated from the 


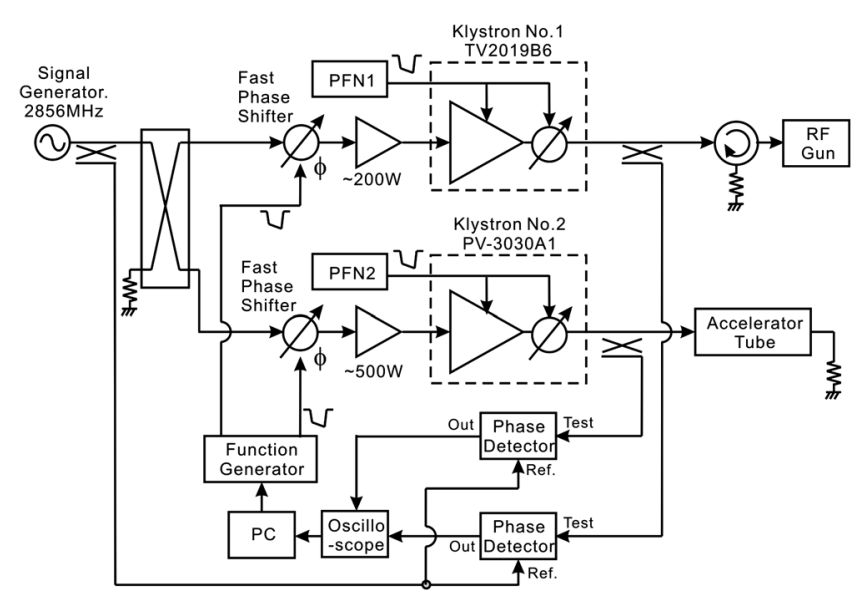

FIG. 3. Schematic diagram of the rf system used to drive the KU-FEL linac. Two fast phase shifters are installed before the klystron driver amplifies.

variation in the electron bunch phase. In this experiment, the $\mathrm{rf}$ signal from a button-type beam position monitor (BPM) installed just before the undulator was used to measure the electron bunch phase. The BPM, developed at KEK [31], has four electrodes that measure the transverse electron position. To measure the bunch phase, rf signals from the four electrodes are combined by a 4-way rf combiner and injected into a phase detector (PDU-NK02N01, NIHON KOSHUHA Co., Ltd.). This method was used to measure the difference between the phase of rf power used to drive the rf gun and the phase of generated electron bunches [32]. The measured bunch phase evolution with different DCD parameters is shown in Fig. 4, where the starting time of DCD is approximately $3 \mu \mathrm{s}$ in the macropulse and the amounts of the phase modulation correspond to cavity detunings of $5.9 \mu \mathrm{m}$ and $9.4 \mu \mathrm{m}$ from the unmodulated condition. With the DCD amount of $9.4 \mu \mathrm{m}$, the highest FEL power could be obtained in the

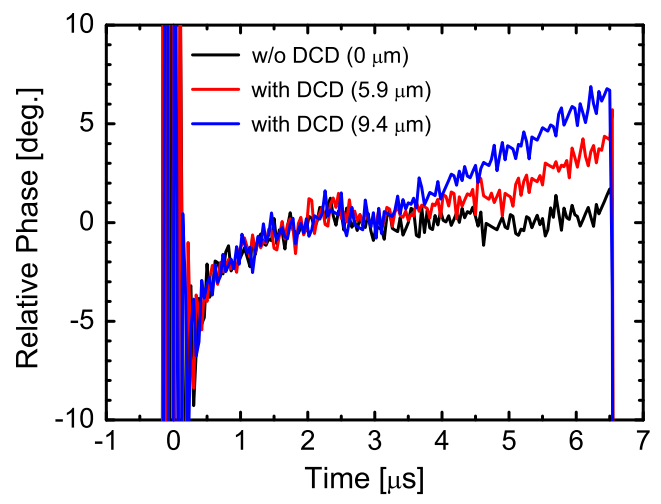

FIG. 4. Measured bunch phase variation with different DCD parameters. The starting time of DCD is approximately $3 \mu \mathrm{s}$ in the macropulse and the amounts of the phase modulation correspond to cavity detunings of $5.9 \mu \mathrm{m}$ and $9.4 \mu \mathrm{m}$ from the unmodulated condition. experiment. The DCD amount of $5.9 \mu \mathrm{m}$ was chosen to be an intermediate condition. Prior to the each extraction efficiency measurement, the FEL power dependence on the relative optical cavity length was measured and the relative optical cavity length was adjusted to have the maximum FEL power at each DCD parameter. Therefore, the optical cavity length was optimized to have the highest extraction efficiency at each DCD parameter. Since we have not implemented a tool to determine the perfectly synchronized cavity length condition, it is difficult to determine the absolute value of the detuning lengths before $3 \mu \mathrm{s}$ in the macropulse. We only can expect the initial detuning lengths from the FEL power dependences on the relative cavity length. The expected detuning lengths before $3 \mu$ s for DCD amount of $9.4 \mu \mathrm{m}, 5.9 \mu \mathrm{m}$ and without DCD were approximately 10,6 , and $2 \mu \mathrm{m}$ from the perfectly synchronized condition, respectively.

\section{Experimental condition}

The arrangement and major specification of the components used in the KU-FEL have been reported elsewhere [14]. The operation conditions of the KU-FEL during the experiment are summarized in Table I. The bunch length $L_{b}$ of KU-FEL has been measured to be $90-180 \mu \mathrm{m}$ (full width at half maximum, FWHM) at an electron beam energy of $27.5 \mathrm{MeV}$ [33]. We expect that the bunch length at an electron beam energy of $27.0 \mathrm{MeV}$ is the same as the reported value. The FEL wavelength is $11.6 \mu \mathrm{m}$, and the slippage length $L_{S}$ is $603 \mu \mathrm{m}$. Under this condition, the bunch length $L_{b}$ is shorter than the slippage length $L_{S}$. Then the superradiant scaling law of a short-bunch FEL oscillator $[25,26]$ is applicable, and high-extractionefficiency operation is possible.

The typical temporal evolution of the bunch charge in a macropulse is shown in Fig. 5. Since a thermionic rf gun is used as the electron source in the KU-FEL, the bunch charge increases from 25 to $55 \mathrm{pC}$ in a macropulse as shown in Fig. 5 due to the back-bombardment effect [34]. Normally, the electron beam energy decreases when the bunch charge of the electron beam increases in an rf linac due to the beam loading effect. In the KU-FEL, the energy

TABLE I. The operation parameters of the KU-FEL during the experiment.

\begin{tabular}{lc}
\hline Electron beam energy & $27.0 \mathrm{MeV}$ \\
Macropulse repetition rate & $2 \mathrm{~Hz}$ \\
Macropulse duration of electron beam & $6.5 \mu \mathrm{s}$ \\
Micropulse repetition rate & $2856 \mathrm{MHz}$ \\
Expected bunch length & $90-180 \mu \mathrm{m}(\mathrm{FWHM})$ \\
Undulator K-Value & 1.34 \\
FEL wavelength & $11.6 \mu \mathrm{m}$ \\
Corresponding slippage length & $603 \mu \mathrm{m}$ \\
FEL cavity roungtrip loss & $\sim 3 \%$ \\
FEL cavity length & $5.0385 \mathrm{~m}$ \\
\hline \hline
\end{tabular}




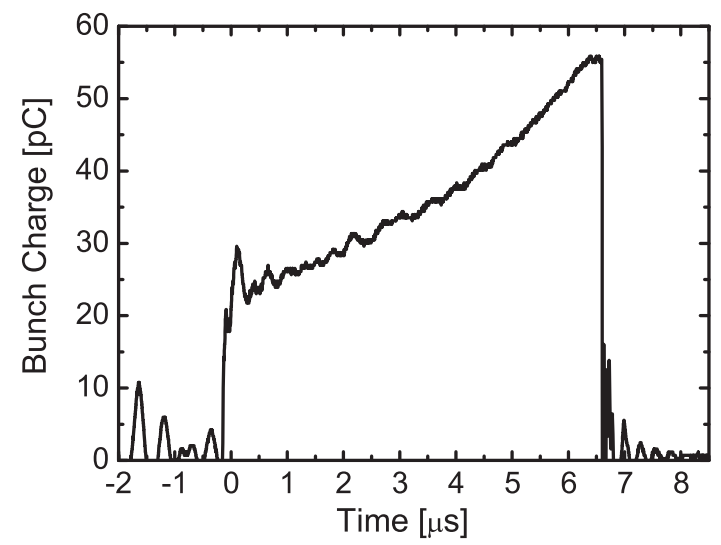

FIG. 5. Typical temporal evolution of the electron bunch charge during a macropulse.
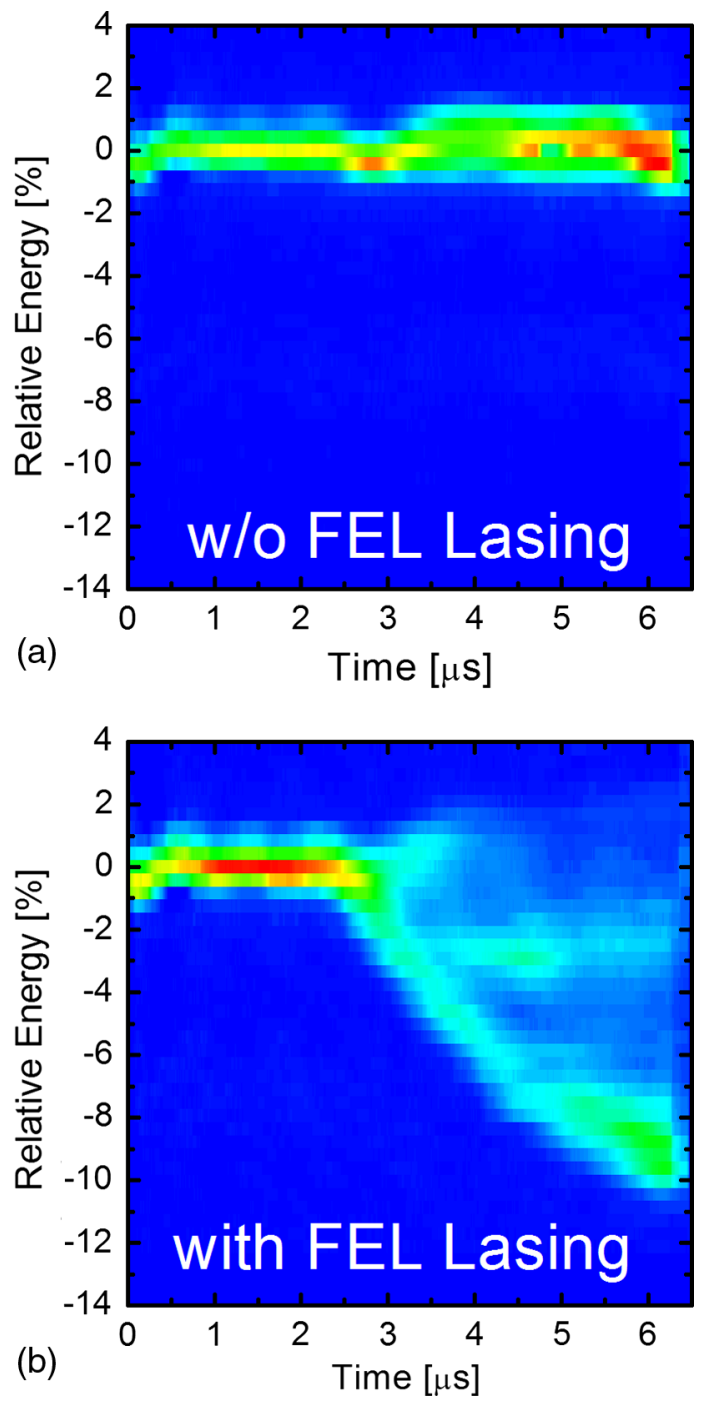

FIG. 6. Measured evolution of the energy distribution of the electron beam with and without FEL lasing for a DCD parameter of $9.4 \mu \mathrm{m}$. (a) without FEL lasing. (b) with FEL lasing. decrease induced by the increase in the bunch charge is compensated by the rf amplitude modulation [35] and the gun cavity detuning method [36]. The roundtrip loss of the FEL optical cavity was evaluated as approximately $3 \%$ from the decaying pattern of the out-coupled laser intensity after the end of the electron macropulse.

\section{EXPERIMENTAL RESULTS}

The measured evolutions of the energy distribution of the electron beam with and without FEL lasing with a DCD parameter of $9.4 \mu \mathrm{m}$ are shown in Fig. 6. The maximum energy loss of the electron with FEL lasing is approximately $9.5 \%$ in this case. At each macropulse time, the average energy of the electron beam is calculated and plotted in Fig. 7(a). The average energy loss induced by FEL lasing is plotted in Fig. 7(b). The maximum average energy loss is approximately $1.5 \mathrm{MeV}$, which corresponds to a relative average energy loss of $5.5 \%$. As explained in the previous section, the relative average energy loss is equal to the extraction efficiency. Therefore, in this case, the extraction efficiency is determined to be $5.5 \%$. The evolutions of the extraction efficiency with different DCD parameters were evaluated in the same way and shown in Fig. 8. As the DCD parameter increases, the FEL pulse
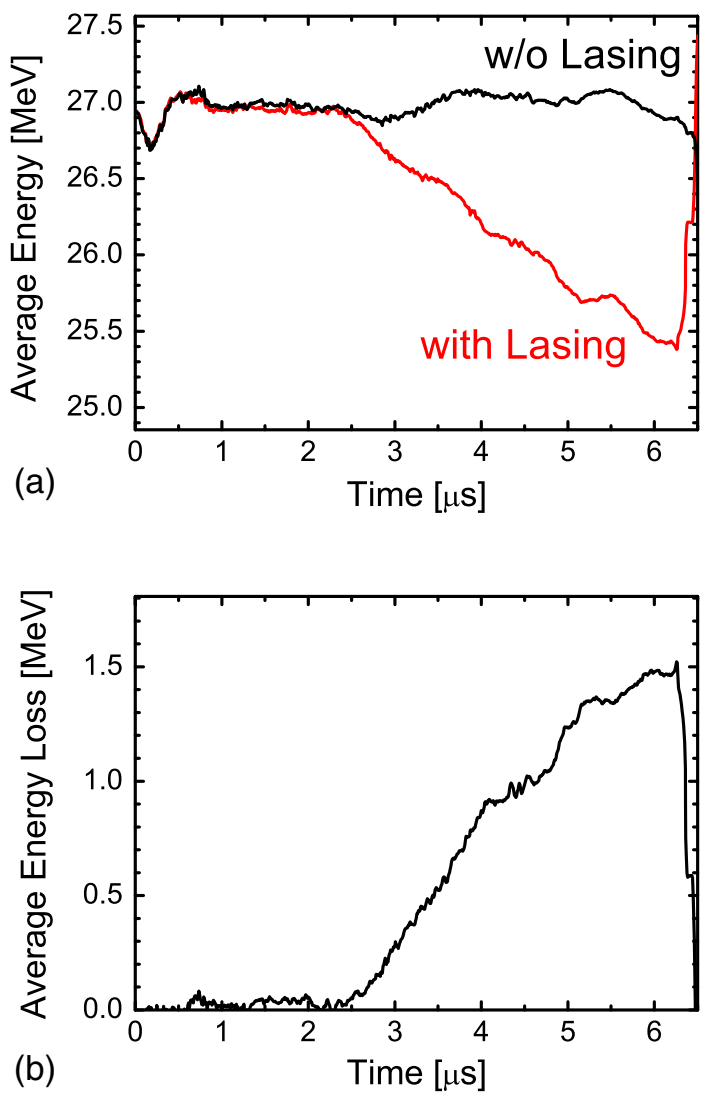

FIG. 7. (a) Evolution of the average energy and (b) evolution of the average energy loss calculated from the measured energy distributions with a DCD parameter of $9.4 \mu \mathrm{m}$. 


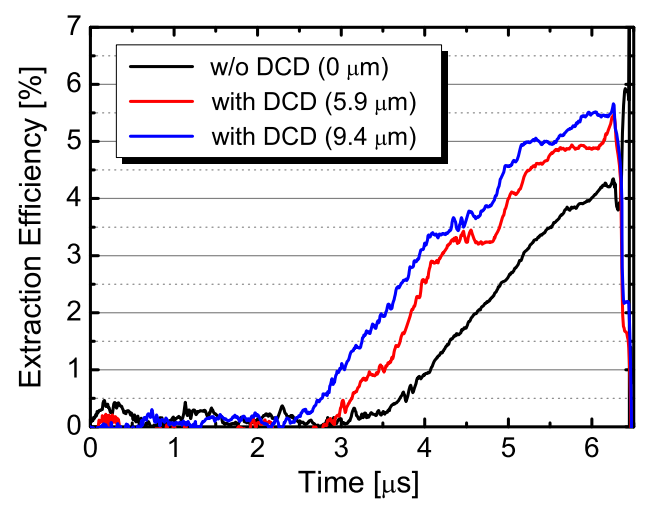

FIG. 8. Evolutions of the extraction efficiency with different DCD parameters evaluated from the evolutions of the energy distribution of the electron beam.

grows earlier and the extraction efficiency at the end of the macropulse increases. Under the DCD parameter of $9.4 \mu \mathrm{m}$, the highest extraction efficiency of $5.5 \%$ was achieved at the end of the macropulse. This value is the highest extraction efficiency ever reported for an FEL oscillator driven by a normal conducting accelerator.

The typical FEL power evolutions with different DCD parameters are shown in Fig. 9(a). By introducing DCD,
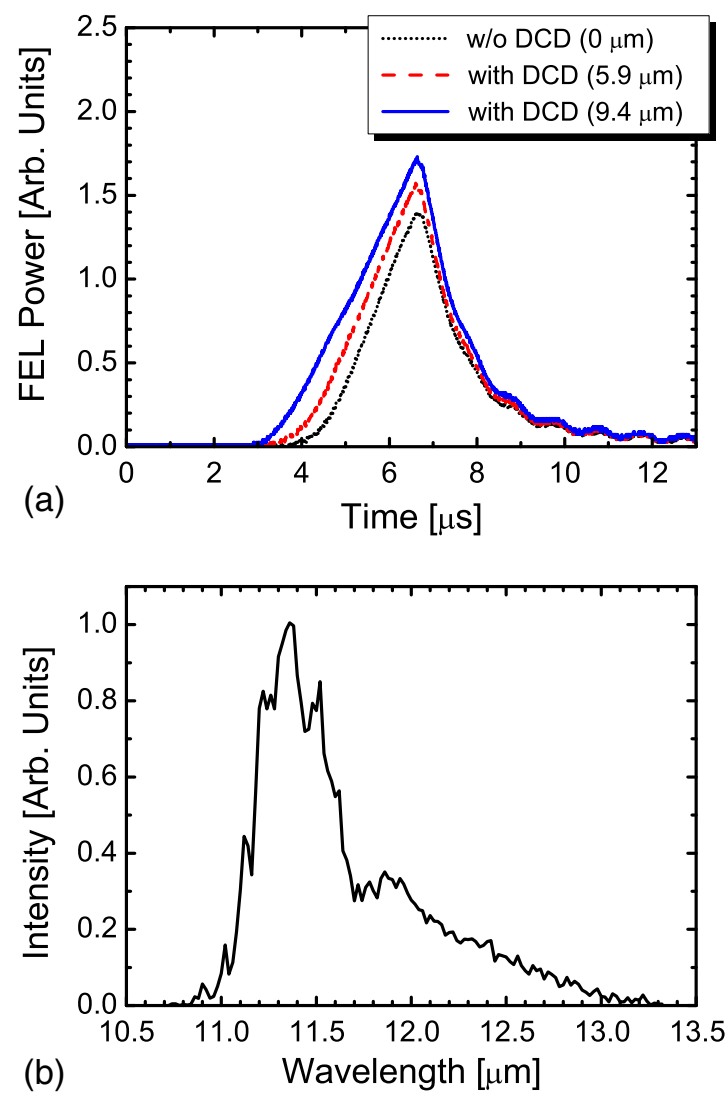

FIG. 9. (a) Typical FEL power evolution with different DCD parameters. (b) Typical FEL spectrum integrated over the macropulse with the DCD parameter of $9.4 \mu \mathrm{m}$. the building up time of the FEL power was shortened and the maximum FEL power was increased. The typical FEL spectrum integrated over the macropulse with the DCD parameter of $9.4 \mu \mathrm{m}$ is shown in Fig. 9(b). The FEL bandwidth is approximately $4 \%$ in FWHM. The spectrum has a long tail in the long wavelength side up to $13.3 \mu \mathrm{m}$.

\section{NUMERICAL SIMULATION}

A numerical simulation was performed to reveal the detailed physics of the high-efficiency lasing observed in the experiment. The simulation code used in this work is a one-dimensional time-dependent code [37]. In the simulation, the electron bunch was assumed to have a charge of $40 \mathrm{pC}$, a rectangular-shaped duration of $1 \mathrm{ps}$ and no energy spread over the macropulse. The FEL parameter, $\rho$, was 0.0034 and the cavity loss was $3 \%$. We assumed a macropulse of $5.7 \mu \mathrm{s}$ for the simulation instead of $6.5 \mu \mathrm{s}$ in the experiment, because the energy and phase were not constant at the leading part of the macropulse as shown in Fig. 4 and the electron bunches at the leading part are considered to have less contribution to the FEL lasing. To simulate the best DCD condition in the experiment, the cavity detuning for the simulation was altered from $9.4 \mu \mathrm{m}$ to $0 \mu \mathrm{m}$ at the 60th round trip, which is $2 \mu \mathrm{s}$ in the macropulse.

First, the evolutions of the energy distribution were examined. The evolution of the energy distribution obtained by the numerical simulation is shown in Fig. 10. In this plot, the relative energy was convoluted with a resolution of $1 \%$, which is the same as the experimental energy resolution. Compared with the experimental result shown in Fig. 6(b), the maximum energy loss in the numerical simulation (7.5\%) is slightly smaller than the experimental result [Fig. 6(b)]. However, the shapes of

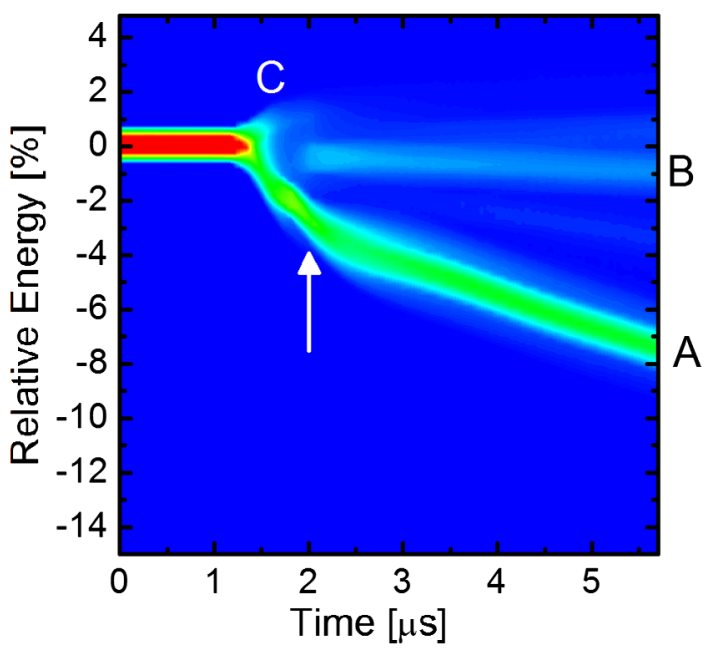

FIG. 10. The evolution of the energy distribution of the electron beam obtained by the numerical simulation under the DCD parameter of $9.4 \mu \mathrm{m}$. The arrow indicates the timing of the cavity detuning condition altering from $9.4 \mu \mathrm{m}$ to $0 \mu \mathrm{m}$. 


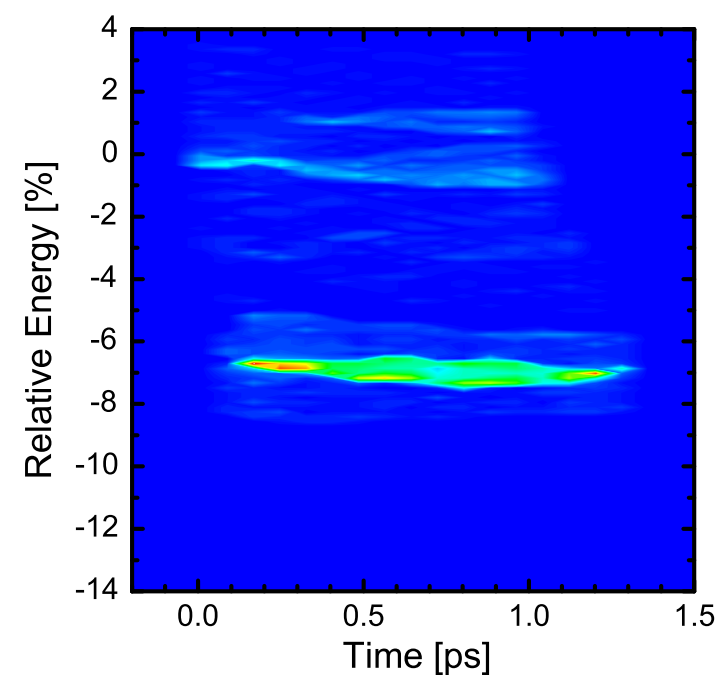

FIG. 11. The longitudinal phase space distribution of the electron bunch obtained by the numerical simulation after the undulator at the end of the macropulse.

the energy evolution map after $2 \mu \mathrm{s}$, which have three splitting structures, in the simulation result are similar to the shapes of the energy evolution map after $3 \mu$ s in the experimental result. Existence of $1 \mu \mathrm{s}$ difference of the DCD timing and the energy evolution map is consistent with the numerical assumption.

The energy evolution maps for the experiment and the simulation have a common structure, with three branches along the macropulse evolution. One branch is the component whose energy is continuously decreasing (A in Fig. 10). Another branch is the component with constant energy (B in Fig. 10). The other branch is the component whose energy is slightly increased and soon disappears (C in Fig. 10). The first branch indicates that a large fraction of the electron beam is trapped in the bucket and efficiently decelerated. This is the reason why a high extraction efficiency was realized in the experiment. The second branch indicates the existence of untrapped electrons in the bucket, but the amount is not very large. The third branch indicates that a small fraction of the electron beam is slightly accelerated in the early phase of the energy extraction.

Figure 11 shows the longitudinal phase space distribution of the electron bunch after the undulator at the end of the macropulse. As shown in Fig. 11, the energy distribution of the electrons is independent of the temporal slices within the bunch. This means that the energy extraction occurs in the entire electron bunch, which contributes to the high extraction efficiency. Figure 12(a) shows the evolution of the energy of electrons with different initial phases in the undulator at the end of the macropulse. Figure 12(b-e) shows the longitudinal phase space distribution of the two wavelength slices at the center of the electron bunch and at different longitudinal position in the undulator. In the
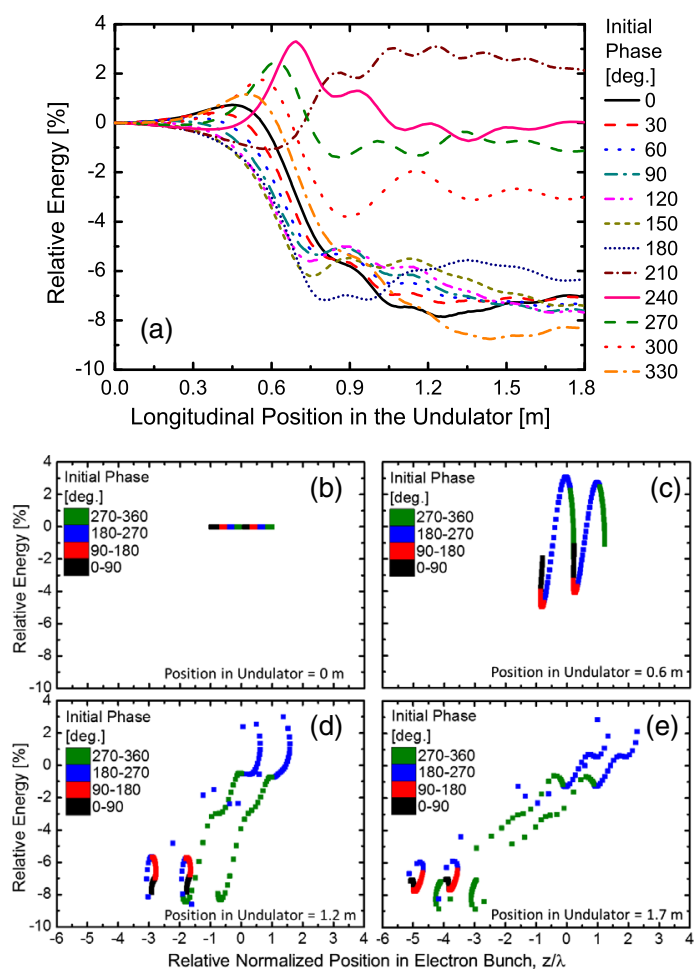

FIG. 12. Evolution of the energy of electrons with different initial phases in the undulator at the end of the macropulse. (a) Variation in relative energy along the undulator. The electrons in one wavelength slice at the center of the electron bunch are plotted. (b-e) phase space plots of the two wavelength slices of the electron beam at the center of the electron bunch and at the different longitudinal position in undulator. The longitudinal position in the undulator for (b), (c), (d), and (e) are $0,0.6,1.2$, and $1.7 \mathrm{~m}$, respectively. The horizontal axis is relative position in the electron bunch normalized by the lasing wavelength $\lambda=11.6 \mu \mathrm{m}$.

simulation, we generate macro-particle whose phases are distributed in the range of 0 to 360 degrees. In Fig. 12, the phase of 158 degrees is corresponding to the bucket center at the undulator entrance. Electrons with an initial phase from 0 to 180 degrees lose their energy with a decrease down to $-8 \%$ at the end of the undulator. These electrons correspond to the first component in the energy evolution map (A in Fig. 10). On the other hand, electrons starting with an initial phase from 210 to 300 degrees do not lose large amount of energy. These electrons correspond to the second component (B in Fig. 10). As shown in Fig. 12, the electrons starting with an initial phase from 240 to 330 are first accelerated and then decelerated. When the FEL electromagnetic field is not strong, the electrons reach the end of the undulator before starting the deceleration. This is the reason for the appearance of the third component (C in Fig. 10). Figure 13 shows the extraction efficiency directly calculated from the numerical result shown in Fig. 10. The maximum extraction efficiency is approximately 5\% in this simulation. This result almost agrees 


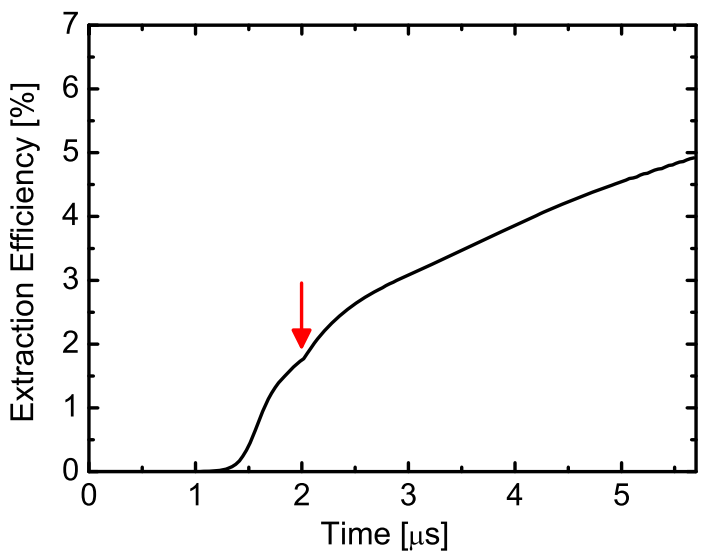

FIG. 13. The evolution of the extraction efficiency directly calculated from the numerical result shown in Fig. 10. The arrow indicates the timing of the cavity detuning condition altering from $9.4 \mu \mathrm{m}$ to $0 \mu \mathrm{m}$.

with the experimental efficiency of $5.5 \%$. We have surveyed DCD parameters, the initial cavity detuning length $\left(5.9 \mu \mathrm{m} \leq d L_{0} \leq 11 \mu \mathrm{m}\right)$ and the timing to alter the cavity detuning condition $(1 \mu \mathrm{s} \leq T \leq 3 \mu \mathrm{s})$, and found the result shown in Fig. 13 is the optimum one to maximize the extraction efficiency at the end of macropulse.

In the present study, the FEL is operated under the condition that the bunch length is shorter than the slippage length, where the lasing behavior is governed by gain parameter integrated over the slippage length [25]. In our simulation, we chose the bunch length of 1 ps corresponding to a half of the slippage length. We confirmed that the extraction efficiency does not increase with shorter bunch as far as the bunch charge is fixed. A shorter bunch contributes to coherent start-up at the early stage of the macropulse $[38,39]$, but the simulation did not include such effect.

\section{CONCLUSION}

The KU-FEL achieved a maximum extraction efficiency of $5.5 \%$ at a wavelength of $11.6 \mu \mathrm{m}$ due to the DCD method [23]. This value is the highest extraction efficiency ever reported for an oscillator-type FEL driven by a normal conducting accelerator. Three DCD conditions (without DCD, $5.9 \mu \mathrm{m}$, and $9.4 \mu \mathrm{m}$ ) were examined to confirm the effectiveness of DCD in increasing the extraction efficiency of an MIR-FEL driven by a normal conducting linac. The measured evolution of the electron energy distribution during a macropulse showed a unique feature of three branches, which was reproduced by the numerical simulation. One of the branches indicates that a large fraction of the electron beam is trapped in the bucket and effectively decelerated. This is the reason why a high extraction efficiency was realized in the experiment. The numerical simulation showed that the entire bunch has similar energy distribution. This means that the energy extraction occurs in the entire electron bunch. The numerical simulation results showed an extraction efficiency of 5\% at the end of the macropulse, which was similar to the experimentally observed efficiency of $5.5 \%$.

The achieved high-extraction-efficiency operation of MIR-FEL will contribute the progress of emerging research areas such as the FEL driven high harmonic generation [40] and the FEL induced periodic surface structure formation [41,42] in MIR region.

\section{ACKNOWLEDGMENTS}

This work was supported by MEXT Quantum Leap Flagship Program (MEXT Q-LEAP) Grant No. JPMXS0118070271.

[1] D. A. G. Deacon, L. R. Elias, J. M. J. Madey, G. J. Ramian, H. A. Schwettman, and T. I. Smith, First Operation of a Free-Electron Laser, Phys. Rev. Lett. 38, 892 (1977).

[2] S. V. Benson, W. S. Fann, B. A. Hooper, J. M. J. Madey, E. B. Szarmes, B. Richman, and L. Vintro, A review of the Stanford Mark III infrared FEL program, Nucl. Instrum. Methods Phys. Res., Sect. A 296, 110 (1990).

[3] R. Prazeres, J. M. Berset, R. Chaput, F. Glotin, D. A. Jaroszynski, and J. M. Ortega, CLIO, an infrared free electron laser facility, Nucl. Instrum. Methods Phys. Res., Sect. B 89, 54 (1994).

[4] D. Oepts, A. F. G. van der Meer, and P. W. van Amersfoort, The free-electron-laser user facility FELIX, Infrared Phys. Technol. 36, 297 (1995).

[5] H. Kuroda, Status of the FEL-SUT project, Jpn. J. Appl Phys. 41, 1 (2002).

[6] H. Horiike, N. Tsubouchi, K. Awazu, M. Asakawa, and M. Heya, Status of the Institute of Free-Electron Laser, Osaka University, Jpn. J. Appl. Phys. 41, 10 (2002).

[7] L. Yonggui, W. Gang, W. Mingkai, Y. Xueping, F. Yaohui, L. Huihua, W. Zihua, Y. Renyong, Z. Junbiao, and X. Lin, Status of the Beijing IR Free Electron Laser facilities, Jpn. J. Appl. Phys. 41, 29 (2002).

[8] P. Michel, F. Gabriel, E. Grosse, P. Evtuschenko, T. Dekorsy, M. Krenz, M. Helm, U. Lehnert, W. Seidel, R. Wünsch et al., First lasing of the ELBE mid-IR FEL, in Proceedings of FEL2004 Conference (Comitato Conferenze Elettra, Trieste, Italy, 2004), pp. 8-13.

[9] E. J. Minehara, Development and operation of the JAERI superconducting energy recovery linacs, Nucl. Instrum. Methods Phys. Res., Sect. A 557, 16 (2006).

[10] S. V. Benson, D. Douglas, G. R. Neil, and M. D. Shinn, The Jefferson Lab free electron laser program, J. Phys. Conf. Ser. 299, 012014 (2011).

[11] N. R. Thompson, D. J. Dunning, J. Clarke, M. Surman, A. Smith, Y. Saveliev, and S. Leonard, First lasing of the ALICE infra-red free-electron laser, Nucl. Instrum. Methods Phys. Res., Sect. A 680, 117 (2012). 
[12] O. A. Shevchenko, V. S. Arbuzov, N. A. Vinokurov, P. D. Vobly, V. N. Volkov, Y. V. Getmanov, Y. I. Gorbachev, I. V. Davidyuk, O. I. Deychuly, E. N. Dementyev et al., The Novosibirsk Free Electron Laser-unique source of terahertz and infrared coherent radiation, Phys. Procedia 84, 13 (2016).

[13] W. Schöllkopf, S. Gewinner, H. Junkes, A. Paarmann, G. von Helden, H. P. Bluem, and A. M. M. Todd, The new IR and THz FEL facility at the Fritz Haber Institute in Berlin, in Proceedings of Advances in X-Ray Free-Electron Lasers Instrumentation III, Prague, Czech Republic, Vol. 9512 (SPIE, Bellingham, Washington, 2015) p. 95121L.

[14] H. Zen, S. Suphakul, T. Kii, K. Masuda, and H. Ohgaki, Present status and perspectives of long wavelength free electron lasers at Kyoto University, Phys. Procedia 84, 47 (2016).

[15] P. Niknejadi, J. M. Kowalczyk, M. R. Hadmack, B. T. Jacobson, I. Howe, S. Kan, S. Smith, E. B. Szarmes, G. Varner, and J. M. J. Madey, Free-electron laser inverseCompton interaction x-ray source, Phys. Rev. Accel. Beams 22, 040704 (2019).

[16] H. Li and Q. Jia, Commissioning and first lasing of the FELiChEM: A new IR and THz FEL oscillator in China, Proceedings of FEL2019 (JACoW Publishing, Geneva, Switzerland, 2019), p. 15, http://accelconf.web.cern.ch/ fel2019/doi/JACoW-FEL2019-MOA07.html.

[17] N. Nishimori, R. Hajima, R. Nagai, and E. J. Minehara, Systematic measurement of maximum efficiencies and detuning lengths at the JAERI free-electron laser, Nucl. Instrum. Methods Phys. Res., Sect. A 483, 134 (2002).

[18] N. Nishimori, R. Hajima, R. Nagai, and E. J. Minehara, Sustained Saturation in a Free-Electron Laser Oscillator at Perfect Synchronism of an Optical Cavity, Phys. Rev. Lett. 86, 5707 (2001).

[19] N. Kroll, P. Morton, and M. W. Rosenbluth, Free-electron lasers with variable parameter wigglers, IEEE J. Quantum Electron. 17, 1436 (1981).

[20] N. Sudar, P. Musumeci, J. Duris, I. Gadjev, M. Polyanskiy, I. Pogorelsky, M. Fedurin, C. Swinson, K. Kusche, M. Babzien et al., High efficiency energy extraction from a relativistic electron beam in a strongly tapered undulator, Phys. Rev. Lett. 117, 174801 (2016).

[21] D. W. Feldman, H. Takeda, R. W. Warren, J. E. Sollid, W. E. Stein, W. J. Johnson, A. H. Lumpkin, and R. B. Feldman, High extraction efficiency experiments with the Los Alamos free electron laser, Nucl. Instrum. Methods Phys. Res., Sect. A 285, 11 (1989).

[22] J. Duris, P. Musumeci, N. Sudar, A. Murokh, and A. Gover, Tapering enhanced stimulated superradiant oscillator, Phys. Rev. Accel. Beams 21, 080705 (2018).

[23] R. J. Bakker, G. M. H. Knippels, A. F. G. van der Meer, D. Oepts, D. A. Jaroszynski, and P. W. van Amersfoort, Dynamic desynchronization of a free-electron laser resonator, Phys. Rev. E 48, R3256 (1993).

[24] R. Prazeres, F. Glotin, and J. M. Ortega, Measurement and calculation of the 'electron efficiency' on the 'CLIO' free-electron laser, Eur. Phys. J. Appl. Phys. 29, 223 (2005).
[25] N. Piovella, P. Chaix, G. Shvets, and D. A. Jaroszynski, Analytical theory of short-pulse free-electron laser oscillators, Phys. Rev. E 52, 5470 (1995).

[26] N. Nishimori, Analytical description of free-electron-laser oscillations in a perfectly synchronized optical cavity, Phys. Rev. E 74, 036502 (2006).

[27] R. Bonifacio, C. Pellegrini, and L. M. Narducci, Collective instabilities and high-gain regime in a free electron laser, Opt. Commun. 50, 373 (1984).

[28] R. Bonifacio, B.W. J. McNeil, and P. Pierini, Superradiance in the high-gain free-electron laser, Phys. Rev. A 40, 4467 (1989).

[29] D. A. Jaroszynski, P. Chaix, N. Piovella, D. Oepts, G. M. H. Knippels, A. F. G. van der Meer, and H. H. Weits, Superradiance in a Short-Pulse Free-Electron-Laser Oscillator, Phys. Rev. Lett. 78, 1699 (1997).

[30] S.-B. Song, S. K. Kim, J. S. Choi, and S. J. Hahn, Dynamic cavity desynchronization in a short pulse freeelectron laser resonator, J. Korean Phys. Soc. 37, 209 (2000); https://www.jkps.or.kr/journal/view.html?uid=4137\&vmd= Full

[31] F. Hinode, H. Hayano, M. Tejima, N. Terunuma, and J. Urakawa, A beam test of button-type beam position monitor for the ATF damping ring, in Proceedings Particle Accelerator Conference (IEEE, New York, 1995), Vol. 4, pp. 2604-2606.

[32] H. Zen, M. A. Bakr, K. Higashimura, T. Kii, R. Kinjo, K. Masuda, K. Nagasaki, and H. Ohgaki, Cavity detuning method to compensate beam energy decrement in thermionic RF gun due to back-bombardment effect, in Proceedings of the International Particle Accelerator Conference, Kyoto, Japan (ICR, Kyoto, 2010), pp. 1725-1727.

[33] N. Sei, H. Zen, and H. Ohgaki, Measurement of bunch length evolution in electron beam macropulse of S-band linac using coherent edge radiation, Phys. Lett. A 383, 389 (2019).

[34] C. B. McKee and J. M. J. Madey, Computer simulation of cathode heating by back-bombardment in the microwave electron gun, Nucl. Instrum. Methods Phys. Res., Sect. A 296, 716 (1990).

[35] T. Kii, Y. Nakai, T. Fukui, H. Zen, K. Kusukame, N. Okawachi, M. Nakano, K. Masuda, H. Ohgaki, K. Yoshikawa et al., Reducing energy degradation due to back-bombardment effect with modulated RF input in S-band thermionic RF gun, AIP Conf. Proc. 879, 248 (2007).

[36] H. Zen, T. Kii, K. Masuda, R. Kinjo, K. Higashimura, K. Nagasaki, and H. Ohgaki, Beam energy compensation in a thermionic RF gun by cavity detuning, IEEE Trans. Nucl. Sci. 56, 1487 (2009).

[37] R. Hajima and R. Nagai, Generation of a Self-Chirped Few-Cycle Optical Pulse in a FEL Oscillator, Phys. Rev. Lett. 91, 024801 (2003).

[38] D. A. Jaroszynski, R. J. Bakker, A. F. G. van der Meer, D. Oepts, and P. W. van Amersfoort, Coherent Startup of an Infrared Free-Electron Laser, Phys. Rev. Lett. 71, 3798 (1993).

[39] B. W. J. McNeil, G. R. M. Robb, and D. A. Jaroszynski, Self-amplification of coherent spontaneous emission 
in the free electron laser, Opt. Commun. 165, 65 (1999).

[40] R. Hajima and R. Nagai, Generating Carrier-EnvelopePhase Stabilized Few-Cycle Pulses from a Free-Electron Laser Oscillator, Phys. Rev. Lett. 119, 204802 (2017).

[41] A. Irizawa, S. Suga, T. Nagashima, A. Higashiya, M. Hashida, and S. Sakabe, Laser-induced fine structures on silicon exposed to THz-FEL, Appl. Phys. Lett. 111, 251602 (2017).

[42] S. Macis, L. Tomarchio, S. Tofani, S. J. Rezvani, L. Faillace, S. Lupi, A. Irizawa, and A. Marcelli, Angular dependence of copper surface damage induced by an intense coherent $\mathrm{THz}$ radiation beam, Cond. Matter 5, 16 (2020). 